\title{
MINIMAL SURFACES BOUNDED BY A PAIR OF CONVEX PLANAR CURVES
}

\author{
WILLIAM H. MEEKS III AND BRIAN WHITE
}

In 1956 M. Shiffman [9] proved several beautiful theorems concerning the geometry of a minimal annulus $A$ whose boundary consists of two closed smooth convex curves in parallel planes $P_{1}$, $P_{2}$. The first theorem stated that the intersection of $A$ with any plane $P$, between $P_{1}$ and $P_{2}$, is a convex Jordan curve. In particular it follows that $A$ is embedded. He then used this convexity theorem to prove that every symmetry of the boundary of $A$ extends to a symmetry of $A$. In the case that $\partial A$ consists of two circles Shiffman proved that $A$ is foliated by circles in parallel planes. Earlier Riemann [7] described, in terms of elliptic functions, all minimal annuli that can be foliated by circles in parallel planes (also see [2] for an analytic description of these surfaces as well as a computer graphics image of one of them). Together these results of Riemann and Shiffman yield a classification of all minimal annuli with boundary consisting of circles in parallel planes.

We shall call a compact minimal surface $M$ stable if, with respect to any nontrivial normal variation fixing the boundary, the second derivative of area is positive. If the second derivative of area is negative for some variation, then $M$ is called unstable. If $M$ is neither stable nor unstable, we will call it almost-stable.

The theorem given below augments Shiffman's first theorem.

Received by the editors November 2, 1989 and, in revised form, September 10, 1990.

1980 Mathematics Subject Classification (1985 Revision). Primary 53A10, 49F10; Secondary 58E12.

The research of the first author described in this paper was supported by research grant DE-FG02-86ER250125 of the Applied Mathematical Science subprogram of Office of Energy Research, U. S. Department of Energy, and National Science Foundation grant DMS-8900285.

The second author was funded by National Science Foundation grants DMS8553231 (PYI) and DMS-8703537. 
Theorem 1. If $\Gamma$ is a pair of smooth convex Jordan curves in distinct parallel planes, then exactly one of the following holds:

1. $\Gamma$ is not the boundary of any connected compact minimal surface, with or without branch points.

2. $\Gamma$ is the boundary of exactly one minimal annulus and this annulus is almost-stable. In this case, $\Gamma$ bounds no other connected compact branched minimal surfaces.

3. $\Gamma$ is the boundary of exactly two minimal annuli; one stable and one unstable. (Perhaps $\Gamma$ is also the boundary of a connected higher genus minimal surface.)

In certain cases it is known that every connected branched minimal surface with boundary $\Gamma$ described in Theorem 1 is actually an annulus. For example, R. Schoen [8] proved that when $\Gamma$ is contained in parallel horizontal planes and is invariant under reflection in two vertical planes, then every branched minimal surface with boundary $\Gamma$ is actually an annulus. Thus, in certain cases, Theorem 1 shows that $\Gamma$ is the boundary of 0,1 , or 2 minimal annuli and no other connected branched minimal surfaces.

The proof of Theorem 1, which we will later outline, is based on an analysis of the Gauss map of a minimal annulus with boundary $\Gamma$ and a geometric approach to calculating the index of a minimal annulus with boundary $\Gamma$. In the proof of Theorem 1 we rely on the description of the space of smooth embedded minimal annuli in $\mathbf{R}^{3}$ as developed in [12] and techniques from global analysis.

Using Theorem 1 and Riemann's classification of minimal annuli foliated by circles, we give a new and simpler proof of Shiffman's second theorem: A minimal annulus bounded by circles in parallel planes is foliated by circles in parallel planes.

An important application of Theorem 1 occurs in proving uniqueness of the solution to a natural free boundary value problem, which we now describe. Suppose $\alpha$ is a Jordan curve in a plane $P_{0}$ and $\Sigma$ is a compact branched minimal surface such that $\partial \Sigma$ consists of $\alpha$ together with a nonempty collection of immersed curves on a parallel plane $P_{1}$. If $\Sigma$ is orthogonal to $P_{1}$ along $\partial \Sigma \cap P_{1}$ and $\partial \Sigma \neq \alpha$, then $\Sigma$ is called a solution of the free boundary value problem for $\alpha$ and $P_{1}$. If, with respect to any nontrivial normal variation of $\Sigma$ that vanishes on $\alpha$, the second derivative of the area functional is positive, then $\Sigma$ is called a stable solution to the free boundary value problem. Similarly, we can define when $\Sigma$ is unstable or almost-stable. 
Theorem 2. Suppose $\Sigma$ is a solution of the free boundary value problem for a smooth convex plane curve $\alpha$ and a plane $P_{1}$ parallel to the plane containing $\alpha$. Then $\Sigma$ is embedded. Furthermore, there exists a unique stable or almost-stable solution $\widetilde{\Sigma}$ to the free boundary value problem for $\alpha$ and $P_{1}$. This surface $\widetilde{\Sigma}$ is an annulus foliated by convex curves in parallel planes.

Theorem 2 is related to Theorem 1 since $\Sigma$ together with its reflected image in $P_{1}$ is a minimal surface bounded by convex curves in parallel planes. The surface $\Sigma$ is a graph over its projection onto $P_{1}$ by [8]. We use this graphical property to prove that when $\Sigma$ is stable or almost-stable then it must be an annulus. Since this annulus together with its reflected image is an annulus with boundary a pair of convex curves in parallel planes, Shiffman's first theorem implies $\Sigma$ is foliated by convex curves.

Recall that a set in $\mathbf{R}^{3}$ is extremal if it lies on the boundary of its convex hull. By generalizing the techniques used to prove Theorem 1 we show:

Theorem 3. The conclusions of Theorem 1 hold under the following weaker hypotheses: $\Gamma$ is a pair of convex planar curves such that $\Gamma$ is not contained in a plane and $\Gamma$ is extremal. Furthermore, all minimal annuli bounded by such $\Gamma$ are embedded.

An immediate consequence of Theorem 3 is the following generalization of Shiffman's symmetry extension theorem. This theorem can fail even for a pair $\Gamma$ of circles when $\Gamma$ is not extremal.

Theorem 4. Suppose $\Gamma$ is a pair of smooth convex planar curves and $\Gamma$ is extremal. Then every symmetry of $\Gamma$ extends to every minimal annulus bounding $\Gamma$.

More generally we consider minimal annuli bounded by smooth Jordan curves on parallel planes. We prove the following nonembeddedness result.

Theorem 5. There exists a pair of smooth Jordan curves in parallel planes that bounds an immersed minimal annulus that is not embedded.

It follows easily from Theorem 5 and the maximum principle that the space $\widetilde{S}$ of smooth immersed minimal annuli bounded by Jordan curves in two fixed parallel planes is not a path connected space. However, we are able to prove: 
Theorem 6. If $S \subset \widetilde{S}$ is the subspace of embedded minimal annuli, then $S$ is path connected. In fact, $S$ is a contractible space.

The theory we develop to prove Theorems 1 and 2 is quite general and probably would be applicable in studying surfaces satisfying other reasonable geometric constraints. For example, motivated by our work, Hoffman, Rosenberg and Spruck [3] have tried a similar geometric approach to describe the set of compact constant positive Gaussian curvature surfaces bounded by a pair of convex planar curves in parallel planes. As in our work, they use the observation that the Gauss map for such a surface is one-toone.

Sketch of the proof of Theorem 1 . Consider the space $\mathscr{M}$ of smooth embedded minimal annuli in $\mathbf{R}^{3}$ with boundary curves in two fixed parallel planes. Let $p: \mathscr{M} \rightarrow \mathscr{C}$ be the associated projection onto the space of pairs of smooth Jordan curves that are contained in the parallel planes. A basic result (see $[11,12])$ is that $\mathscr{M}$ and $\mathscr{C}$ are smooth infinite dimensional manifolds and $p$ is a proper Fredholm map of index zero.

In order to apply the powerful methods from global analysis to prove Theorem 1, one first needs some specific information about the "index of stability" of a minimal surface. The index of stability is the number of negative eigenvalues (counting multiplicity) of the second derivative of the area functional. Since the second derivative of area is positive for any variation of a stable minimal surface, a stable minimal surface must have index zero.

Let $A$ be a minimal annulus with convex boundary $\Gamma \in \mathscr{C}$. To get detailed information on the index of stability of $A$, we apply: a classical result of Schwarz [1]. If $M$ is a compact minimal surface and the Gauss map $G$ is one-to-one on the interior of $M$, then the index of stability is equal to the index of $\Delta+2$ on $G(M)$, where $\Delta$ is the Laplace-Beltrami operator on $S^{2}$. A straightforward application of the open mapping theorem shows that the Gauss map of $A$ is one-to-one on the interior of $A$. With respect to $\Delta+2$, a proper subdomain of $S^{2}$ can have at most one nontrivial eigenfunction with eigenvalues less than or equal to zero. Therefore Schwarz's theorem implies that the index of $A$ is at most 1 and if $A$ has index 1 , then the second derivative of the area functional has no zero eigenvalues; i.e., the projection $p$ has trivial kernel. In particular $A$ is either stable, almost-stable or unstable of index 1 . 
A surface $\Sigma \in \mathscr{M}$ is called a regular point of $p: \mathscr{M} \rightarrow \mathscr{C}$ if the derivative $p_{*}: T_{\Sigma} \mathscr{M} \rightarrow T_{\partial \Sigma} \mathscr{C}$ has trivial kernel; and $\alpha \in \mathscr{C}$ is a regular value of $p$ if $p^{-1}(\alpha)$ consists of regular points. By the Sard-Smale Theorem [10], almost all $\alpha \in \mathscr{C}$ are regular values of $p$ in the sense of Baire category. When $\alpha=\Gamma$ is a pair of convex curves, we have already identified the nonregular points of $p^{-1}(\Gamma)$ as those annuli that are almost-stable. Thus for generic $\Gamma$, every annulus with boundary $\Gamma$ is unstable of index 1 or is stable. From this point on, we will make the additional assumption: $\Gamma$ is a regular value of $p$.

Since $p: \mathscr{M} \rightarrow \mathscr{C}$ is a proper map, for $\alpha$ a regular value, $p^{-1}(\alpha)$ consists of a finite number of minimal annuli. This properness property of $p$ has been used by White [12] to prove that the number of odd index annuli in $p^{-1}(\alpha)$ is equal to the number of even index annuli. Since the minimal annuli bounded by $\Gamma$ have index 0 or 1 , the number of stable examples equals the number of unstable ones. In particular, if $\Gamma$ is the boundary of more than one unstable minimal annulus, then it bounds at least two stable minimal annuli.

Suppose $\Gamma$ is the boundary of a connected minimal surface $\Sigma$. Then using $\Sigma$ as a barrier, the Geometric Dehn's Lemma of Meeks-Yau [6] shows that $\Gamma$ is the boundary of a least-area minimal annulus $A$ that lies "outside" $\Sigma$. Applying the properness property of $p$ and the Meeks-Yau barrier argument, we prove that there exists a stable or almost-stable minimal annulus $\mathscr{A}$ that is outermost in the sense that every compact minimal surface $\Sigma$ with $\partial \Sigma=\Gamma$, lies inside the ball with boundary $\mathscr{A}$ union the planar disks bounded by $\Gamma$.

Suppose $\Gamma$ is the boundary of another stable minimal annulus $A$ inside $\mathscr{A}$. It follows (by e.g., a minimax argument) that there is an unstable minimal annulus $A_{u}$ with boundary $\Gamma$ such that $A_{u}$ lies geometrically between $\mathscr{A}$ and $A$, and $A_{u}$ is never tangent to $\mathscr{A}$ or $A$ along $\Gamma$. Since $A_{u}$ lies outside $A$, a simple geometric argument shows that $N\left(A_{u}\right) \subset N(A) \subset S^{2}$, where $N$ denotes the normal maps for these surfaces. Since larger domains of $S^{2}$ have smaller first eigenvalue with respect to $\Delta_{S^{2}}+2$, Schwarz's theorem implies $A_{u}$ must be stable (since $A$ is stable). This contradiction shows that $A$ cannot exist. Hence $\Gamma$ is the boundary of no connected minimal surface or $\Gamma$ is the boundary of exactly two minimal annuli, one stable and one unstable. 
The proofs of Statement 3 of Theorem 1, in the case $\Gamma$ is not a regular value of $p$, and of Statement 2 in Theorem 1 require some further arguments.

All the results described here will appear in [4] and [5].

\section{REFERENCES}

1. J. L. Barbosa and M. do Carmo, On the size of a stable minimal surface in $\mathbf{R}^{3}$, Amer. J. Math. 19(8) (1976), 515-528.

2. M. Callahan, D. Hoffman, and W. H. Meeks III, Embedded minimal surfaces with an infinite number of ends, Invent. Math. 96 (1989), 459-505.

3. D. Hoffman, H. Rosenberg, and J. Spruck, personal communication.

4. W. H. Meeks III and B. White, Minimal surfaces bounded by convex curves in parallel planes, Comment. Math. Helv. (to appear).

5. _ - The space of minimal annuli bounded by an extremal pair of planar curves, preprint.

6. W. H. Meeks III and S. T. Yau, The existence of embedded minimal surfaces and the problem of uniqueness, Math. Z. 179 (1982), 151-168.

7. B. Riemann, Ouevres mathématiques de Riemann, Gauthiers-Villars, Paris, 1898.

8. R. Schoen, Uniqueness, symmetry, and embeddedness of minimal surfaces, J. Differential Geom. 18 (1983), 791-809.

9. M. Shiffman, On surfaces of stationary area bounded by two circles, or convex curves, in parallel planes, Ann. of Math. (2) 63 (1956), 77-90.

10. S. Smale, An infinite dimensional version of Sard's theorem, Amer. J. Math. 87 (1965), 861-866.

11. F. Tomi and A. J. Tromba, Extreme curves bound an embedded minimal surface of disk type, Math. Z. 158 (1978), 137-145.

12. B. White, New applications of mapping degrees to minimal surface theory, J. Differential Geom. 29 (1989), 143-162.

Department of Mathematics, University of Massachusetts, Amherst, MASSACHUSETTS 01003

Department of Mathematics, Stanford University, Stanford, CaliforNIA 94305 